\title{
Home away home: Adjustment experience of Bangladeshi immigrants in the Netherlands
}

\author{
K. S. Farid \\ Department of Rural Sociology, Bangladesh Agricultural University, Mymensingh-2202, Bangladesh \\ E-mail: ksfarid@gmail.com
}

\begin{abstract}
Adjustment into a new culture is of crucial importance for the immigrants to lead a meaningful life in that culture. The present study investigates the adjustment experiences of Bangladeshi immigrants in the Netherlands by exploring and describing the nature of psychological, socio-cultural, and economic adjustment. Participants were 14 Bangladeshi immigrants aged between 29 and 62 years who were selected through snowball sampling technique, were directly or indirectly related with restaurant business and had been residing in the Netherlands for at least five years. A qualitative approach to data collection and analysis was employed in order to get insights from the study participants. For collecting data, in-depth interviews were conducted among the study participants. Data analysis was done with the use of MAXQDA qualitative data analysis software. Examination of the interview transcripts revealed that the immigrants are psychologically happy with their well-being, as almost all of their expectations have been fulfilled despite some problems of cultural differences. Bangladeshi immigrants maintain socio-cultural relationship with the people sufficient to lead normal daily lives as they do hardly face any problem. However, there interaction with Dutch is limited. Though they are not completely happy with their busy lives in the Netherlands, they feel better here compared to Bangladesh. They are economically adjusted because of the fulfilment of their economic expectations. Several practical implications were elicited from the research including a need for programmes that promote immigrants' integration with the mainstream of the Dutch society by encouraging the broadening of social relationship.
\end{abstract}

Keywords: Socio-cultural adjustment, Psychological adjustment, Economic adjustment, Bangladeshi immigrants

\section{Introduction}

Proper adjustment of the immigrants with the host society is a crucial issue and adjustment experience of these immigrants poses major challenge to politicians, practitioners, and researchers. Immigration to a new country is a stressful life event that is associated with cultural shock and requires personal, economic, and social-cultural changes. There is growing consensus on the need to promote immigrants' integration into the labour market and society. Integration of immigrants, however, requires immigrants to adjust psychologically, socio-culturally and economically into the host society (Blunt, 2007; Moghaddas et al., 2006; and Zlobina et al., 2006). Therefore, there is an increasing need to understand and identify the nature of adjustment of immigrants; whether they are adjusted or not, and how much they are adjusted in the host country. Various studies conducted in this regard found mixed result. Majority of the Sudanese refugees resettled in Tasmania described themselves as happy with their current lives in Tasmania, and reported being involved in social activities. In comparison to life in Sudan, they saw Australia as a peaceful and safe place to live (Shakespeare-Finch and Wickham, 2010). Socio-cultural adjustment along with psychological adjustment has been conceptualized as a major dimension of intercultural adjustment in the study of Zlobina et al. (2006). Socio-cultural adaptation was conceptualized in terms of the problem of inter-cultural communication such as cultural knowledge or coping with the language, and of successful resolving of practical problems that imply interaction with host. Findings showed that social difficulty of the immigrants decreased over time, which helped them to adjust in the host society. Phalet and Hagendoorn (1996) identified that Turkish immigrant youth with a collectivistic value orientation have fewer adjustment problems in Belgium and they have better adjustment experience thereof. Again, cultural distance adds to external adjustment problems and weakens their adjustment process in the host country. 
Saikia (2002) found that almost half of the Bangladeshi immigrants in India are not adjusted psychologically and more than one-third are not socially. Most of the illegal immigrants are weakly adjusted or not adjusted. Again, immigrants who are strongly adjusted socially are not necessarily strongly adjusted psychologically because of the fear of being foreigner and of fear of communal riots. However, most of the immigrants' adjustment process is in transition type and it is close to non-adjusted type. Therefore, immigrants' adjustment into a new setting is a complex issue, which needs to be addressed and studied properly. But the process of socio-cultural and psychological adjustment of the immigrants in the host society has been a neglected part in the contemporary migration research (Zlobina et al., 2006). Though many researchers have turned their attention to the adjustment process of immigrants in recent years, qualitative research on adjustment experience of immigrants in the host society is of crucial importance. However, there is hardly any study regarding adjustment process of Bangladeshi immigrants in the Netherlands. The present study was, therefore, an attempt to explore and describe the nature of adjustment of Bangladeshi immigrants in the Netherlands based on psychological adjustment, socio-cultural adjustment, and economic adjustment.

\section{Materials and Methods}

A qualitative research design was adopted in this study in order to explore and describe the nature of adjustment of immigrants. Population of the present study consisted of the immigrants of Bangladeshorigin who had been living in the Netherlands for at least five years, and are directly or indirectly related to the restaurant business. Primarily immigrant communities were identified through personal communication. A snowball sampling technique was adopted to select the participant for the current study. Firstly, contact was made with the potential participants. A list of participants who wished to participate in the study was made then. Twenty immigrants were identified through snowball. All the immigrants are Muslim. Among them, 18 immigrants either own or work in the restaurant of whom two refused to participate in the study. Among the rest 16 participants, data were collected from 14 immigrants, which was determined based on data saturation. Therefore, participants of this study are 14 Bangladeshi immigrants who have immigration status eight (8) of whom are the owner of the restaurant and the other six (6) works in the restaurant. There are two immigrants aged less than 30 years, six are between 30-45 years, five are 46-60 years category and the rest one is more than 60 years. The youngest participant is 29 years old and the age of oldest participant is 62 years. The participants migrated to the Netherlands at young age. 11 participants migrated from 20 to 30 years of age, two participants were less than 20 years and another one was higher than 30, with a lowest of 19 and highest of 37. The highest 6 of the participants completed more than 14 years of schooling followed by four from 10 to 12 years and two each for lower than 10 years and from 13-14 years of schooling, respectively. Moreover, the lowest and highest years of schooling completed by the study participants are 6 and 17 years. Among the 14 participants, 11 are married and the rest have official Dutch girl friend. One participant's wife is Dutch citizen and another one is Philippines. Almost all of them have one or two children except the participants those have girl friends.

In-depth interviews were conducted with the participants to collect necessary data at their convenient. Participants were formally interviewed in a single sitting lasted between 40 and 60 minutes depending on the situation of the respective interview. Interviews was conducted in Bengali, the mother tongue of Bangladeshi, and tape-recorded with the prior consent of the participants. All recorded interviews were turned into verbatim transcripts. The transcription was produced in Bengali and then translated into English by the researcher himself. All identifiers from the transcripts were then removed in order to preserve the anonymity of the participants. After data preparation, a set of deductive codes (based on interview guide and literature review), inductive codes (issues raised by the participants) and in-vivo codes (specific phrases and metaphors used by participants) was produced. Then coding was done by labelling all the data using all listed codes through careful reading and then identifying sections of data relevant to each code. A thick description of each code was developed through careful reading of texts of each code. A cross-case comparison of codes was made to identify patterns and associations in the data 
and to clarify issues that make each issue or code distinct from others. Then codes with similar characteristics were identified and grouped into meaningful categories. Concepts were then made based on the relationships between the categories. These concepts were developed taking into consideration of specific research questions. Data were analyzed with the use of MAXQDA qualitative data analysis software.

Ethical issues are very much important in doing socio-demographic research (Hennink et al., 2011), which were considered carefully in this study. The participation in the research process was voluntary. The process of data collection was done based on informed consent of the respondents. They were fully informed about the objectives and the procedure involved in the present research. Assurance was also given to the participants that the individual information will not be presented in the paper. Data were collected in such a manner so that the participants are not mentally or psychologically injured during interview. Probing during interview was used in such way that will not injure respondents' self-esteem. The privacy of the participants was ensured properly. They were assured that identifying information will not be made available to anyone and their information will remain confidential throughout the whole period of study and identifying characteristics will be eliminated after data entry.

\section{Results and Discussion}

Nature of adjustment of Bangladeshi immigrants is presented in this chapter based on the results of indepth interviews. Nature of adjustment is explained here in terms of three broad categories: psychological adjustment, socio-cultural adjustment, and economic adjustment.

\section{Psychological adjustment}

Nature of psychological adjustment of the immigrants is explained in this section in terms of immigrants' feeling about well-being, feeling of being foreigner, perceived sense of discrimination, perceived cultural distance, sense of belongingness, and level of expectation and satisfaction. Personal sense of well-being is an important attribute of psychological adjustment of an individual. The term 'well-being' is used here as synonymous of Bengali word 'sukh-sacchondo' (happiness). It is measured in terms of the participants' feeling about whether they are better off now or not regarding their personal, family, social and economic life. When the participants were asked about their views of their well-being, they compared their present life in the Netherlands with the life they led in Bangladesh and with another individual of their age living in Bangladesh. In comparing so, the participants feel that they are better now. They also feel better thinking that they are better in position compared to some Dutch and some other fellow immigrants.

Psychological adjustment is associated with the degree of integration of the immigrants with the host society. The lesser they have feeling of being foreigner, the higher the extent of their psychological adjustment and vice versa. Almost all the immigrants had some sorts of feeling that they are foreigner during the early part of their stay in the Netherlands. Nevertheless, though the immigrants are being able to overcome this problem by the course of time, this type of feeling exist until now in some cases. Some of them observed that some Dutch do not like them, which creates adjustment problems from psychological points of view. Moreover, immigrants are anxious of the possibility of formulation of antiimmigration law. Immigrants face problems in adjusting psychologically when they have the sense of discrimination. Though most of the participants mentioned that they do not feel any kind of discrimination, a few of them mentioned that they are being discriminated.

The Bangladeshi immigrants are leading a very different life in a very different culture with differences in language, religion, weather, food habits, social life, family life, economic system, gender relations, etc. Religious and cultural differences played negative role in case of adjustment of Bangladeshi immigrants. Cultural differences act as a real problem for children of the immigrants. Immigrants are suspicious of what culture their children should learn and adopt. If their children do not adopt Dutch culture, they might face problems in the school. It becomes difficult for them to establish relationship with their peer. However, sometimes the participants feel fear when print and electronic media broadcast anti-Islam 
programmes. During the informal discussions after in-depth interview session, the participants mentioned that Dutch media sometimes spread hatred against Islam, they mentioned about Geert Wilders' short film Fitna. Mental satisfaction along with the fulfilment of expectation is very much important for the psychological adjustment of the participants. Almost all of the participants reported that their expectations have been fulfilled and they are satisfied with their present life. The participants are very much satisfied with social security measures of the Dutch government. In comparing with the life of Bangladesh, they feel that they are leading better life in the Netherlands. They are now not only able to take care of themselves and their family but also to support other needy people back home. They have mental strength that they have no problem now.

Usually immigrants tend to feel multiple senses of belongingness. Along with the sense of belongingness to their home country, they feel sense of belongingness to the host country. Almost all participants feel dual sense of belongingness in their mind. It is impossible for them not to feel belongingness to their home country. Most of the participants do not have any concrete plan of settlement. Some of them mentioned that they are already settled in this country and they want to stay here. They do not want to face insecurity going back to Bangladesh. Whereas, some other immigrants think they will go back to Bangladesh in their old age. Decision of future settlement also depends on the career of children. The participants feel that their children are secured here; they have good prospects in this country. Therefore, they do not want to return to Bangladesh for the sake of their children.

From the discussions, it can be said that the Bangladeshi immigrants show a mixed pattern in terms of their psychological adjustment in the Netherlands. In some perspectives, they are psychologically adjusted; but in some other aspects, they are not. For example, they feel that they are in a better position now and almost all of their expectations have been fulfilled. They are satisfied with their personal, family, social, and economic life. Their life is better in the Netherlands compared to Bangladesh. In general, they do not feel that they are being discriminated. In some instances, cultural differences create problems not for themselves but for their children. Though there are very few instances of racial discrimination in the Netherlands, Bangladeshi immigrants have fear of being discriminated racially in some cases.

\section{Socio-cultural adjustment}

Dutch 'multicultural model' of immigrant integration emphasize on cultural emancipation of immigrant minorities for their integration into Dutch society. Socio-cultural adjustment of the immigrants is predominantly related with the behavioural and cognitive elements of cultural learning in order to perform in a new culture properly (Duyvendak and Scholten, 2011). It depends on how positively immigrants perceive their lives in the new society, their ability to do normal daily activities, nature of relationship and interaction they maintain with others, and nature of participation in various social programmes. In almost all cases, participants mention their lives as a busy life. This is mostly true for the owners of the restaurant. Their life is a routine life and they are not happy with this routine life. In some cases, those who work in the restaurant do not feel good. They are not mentally happy. Sometimes they feel that they are fine with their job thinking about their secured life from social and economic point of view. However, in some cases they feel guilty thinking that they are working in the restaurant, which is not respectable in their home country.

For some Bangladeshi immigrants life is nothing but to work. They do have very little social life. Their life is only to work in the restaurant, earn living, return to house, sleep there, wake up next day, and start work again. They are in vicious circle of 'working-eating-sleeping-working'. In their words, "kaj-khaoyaghum-kaj" (work, eat, sleep and work). The owners have to manage all types of activities related to the restaurant business along with their personal and household activities whereas; the workers do personal and family related activities besides working in the restaurant. The results of the in-depth interviews show that the owners as well as the workers of the restaurant are doing well in doing their normal daily activities and they are not facing any problem in doing so. Establishing relationship with the surrounding environment is a prerequisite for proper adjustment in a new culture. Immigrants perceived that it was easy to find and establish relationship with the host before two decades as the Dutch were supportive to 
the immigrants during that time. Recently attitudes toward immigrants are changing negatively. Now- adays, Dutch consider immigrants as problem. Participants in this study mentioned that Dutch do not like immigrants anymore, which are creating mental pressure for them. As a result, it is somewhat difficult for them to maintain relationship with the host society. Moreover, it was very much difficult in some instances for the participants to establish new relationship with Dutch due to the differences in culture, language, and food habit.

The participants mainly interact with other Bangladeshi immigrants. They have in general little contact with other fellow immigrants from other countries. Their interaction with Dutch is limited. It is interesting that participants' wives and children have more interaction with Dutch than that of themselves. Most of the cases they have some Dutch family friends. This relationship is established based on the friendship of the children in the school. Some immigrants, in turn, mentioned that they interact with all types of people. A few also mentioned that their interaction is limited with Bangladeshi immigrants. In almost all cases, the neighbours of the Bangladeshi immigrants are Dutch. In very few cases their neighbour are fellow immigrants from other countries and they do not have Bangladeshi neighbour. As a result, they have to keep communication with Dutch. However, their contact with Dutch is limited, most of the cases not more than 'hi-hellos'. They do have very limited home visits and phone calls. They visit each others' house occasionally such as during any party arranged. However, this is not also so common. Nevertheless, most of the Bangladeshi immigrants have good relationship with at least one Dutch or a Dutch family. They have continuous communication with each other. They invite them, visit each other's house, greet in various occasions, for example, birthday, anniversary, New Year etc. They help each other in case of necessity.

Socio-cultural relationship of an individual with other individuals of the society is an important determinant of socio-cultural adjustment, which depends on the extent of celebration of various types of occasions. Bangladeshi immigrants do not usually celebrate and participate in many programmes regardless of religious or national. Sometimes they celebrate some religious programmes personally and invite some of their friends. They are in general, do not actively celebrate Dutch religious and national occasions, but try to enjoy the occasions Moreover, the Bangladeshi immigrants find it difficult to celebrate Bangladeshi occasions for not having any official vacation during those days.

The above-mentioned discussions suggest that the Bangladeshi immigrants do not have wide-ranged socio-cultural relationships with the community and the society. However, they maintain socio-cultural relationship, which is somewhat sufficient for leading a meaningful socio-cultural life as they do hardly face any problem at present. Though they are not happy with their present busy lives in the Netherlands in some cases, they feel better here compared to Bangladesh. Primarily it was somewhat difficult for them to establish new relations with other people of Dutch society. Nevertheless, they have overcome these difficulties in the course of time. The Bangladeshi immigrants generally interact with all types of people such as, fellow Bangladeshi immigrants, other immigrants from other countries, and the Dutch people. However, their interaction with Dutch is not wide spread. Nevertheless, they do not consider it as problematic.

\section{Economic adjustment}

The study of economic adjustment of immigrants is highly relevant as immigrants are often found to hold economically disadvantaged positions in host societies (Vroome and Tubergen, 2010). It is also relevant as the Bangladeshi immigrants mainly immigrated for the economic pursuit. Among the 14 participants of this study, eight are owner of the restaurant and the rest six works in the restaurant owned by Bangladeshi. The important matter is that by establishing restaurant business, Bangladeshi immigrants become entrepreneurs. They not only provide employment opportunity to themselves and some other fellow Bangladeshi immigrants, but also provide job to some Dutch and some other immigrants of other countries. Moreover, the participants feel that becoming owner is a respectful position in Dutch society. This restaurant business gives them the honour in this society. 
Becoming economically independent is the most important reason of immigration of Bangladeshi immigrants. Satisfaction about economic position is crucial for not only their economic adjustment but also psychological and socio-cultural adjustment as it is their main goal. Almost all of the participants feel that their economic expectations have been fulfilled, and they are satisfied with their current economic position. They also feel that they are completely economically independent. It can be said that economic adjustment is more important for Bangladeshi immigrants than that of socio-cultural and psychological adjustments. Being economically independent is an important criterion for proper economic adjustment. It is apparent that the immigrants are very much satisfied with their economic achievement. This is because; almost all of their economic expectations have been fulfilled. Therefore, they are economically adjusted.

\section{Conclusion}

This study examines the nature of psychological, socio-cultural, and economic adjustment of the Bangladeshi immigrants. Participants have positive perceptions about their well-being, i.e., they feel that they are in better off condition now compared to their lives in Bangladesh. Feeling of being foreigner is not so strong for the Bangladeshi immigrants. However, they feel that they are being discriminated in very few cases. Cultural distances become a problem for their children in some instances. What is important is that they feel that their expectations have been fulfilled in almost all cases and they are very much satisfied with their present life. Though the participants were passing very busy life, they are happy with the way they lead their lives in the Netherlands as they are in general not facing any problem. Generally, they are maintaining good relationship with all types of people in the Dutch society, though their interaction is limited with the Dutch in some cases. Therefore, despite a few limitations, Bangladeshi immigrants are more or less adjusted from socio-cultural point of view. Immigrants of Bangladesh, however, are adjusted economically more than that of psychological and socio-cultural adjustment. Their economic expectations have been fulfilled in almost all cases. They are economically independent and they are very much satisfied with their economic position. In conclusion it can be said that economic consideration is most important for Bangladeshi immigrants. The extent of maintaining social relationship is not so important for them. Their psychological well-being is mainly related with their economic wellbeing. It seems that they can compensate their socio-cultural life in the pursuit of economic well-being. Based on the discussion of the results, this study recommends taking effective initiatives to improve the community relationship of the immigrants, broadcasting the contributions of the immigrants through mass media so that the Dutch think positively about immigrants and combating discrimination by ensuring equal rights and equal opportunities for everyone, irrespective of ethnic and racial origin.

\section{References}

Blunt, A. 2007. Cultural geographies of migration: Mobility, transnationality and diaspora, Progress in Human geography, 31(5):684694.

Duyvendak, J.W. and Scholten, P.W.A. 2011. Beyond the Dutch 'multicultural model': The coproduction of integration policy frames in the Netherlands. International Migration and Integration, 12:331-348.

Shakespeare-Finch, J. and Wickham, K. 2010. Adaptation of Sudanese refugees in an Australian context: Investigating helps and hindrances. International Migration, 48(1):23-46.

Hennink, M., Hutter, I. and Bailey, A. 2011. Qualitative Research Methods. London, SAGE Publications Ltd.

Moghaddas, A.A., Amiri, A.M. and Rahimi, A. 2006. The process of adjustment/acculturation of first and second generation of migrants: The case of nomad Qashquee Turks immigrants in Shiraz-Iran. Paper presented at European Population Conference held from 21-24 June 2006 at Liverpool, UK.

Phalet, K. and Hagendoorn, L. 1996. Personal adjustment to acculturative transitions: The Turkish experience. International Journal of Psychology, 31(2):131-144.

Saikia, U. 2002. Adjustment process of immigrants in the midst of ethnic conflict: A case of Bangladeshi immigrants in India. Paper presented at IUSSP Regional Population Conference held from 10-13 June 2002 at Bangkok, Thailand.

Vroome, de T. and Tubergen, van F. 2010. The employment experience of refugees in the Netherlands. International Migration Review, 44(2):376-403.

Zlobina, A., Basabe, N., Paez, D. and Furnham, A. 2006. Socio-cultural adjustment of immigrants: Universal and group-specific predictors. International Journal of Intercultural Relations, 30: 195-211. 